\title{
Research on the Solar collector thermoelectric power generation
}

\author{
Huasheng Wang
}

North China Electric Power University (Baoding), Baoding. 071000, China

Keywords: solar, thermoelectric power generation, thermal optimization

\begin{abstract}
In the modern period of rapid economic growth, energy shortage is serious, the new type of solar renewable green energy, has the character of economy, clean environment, the advantages of large reserves. This paper puts forward a new kind of solar collector thermoelectric power generation device. Combined with the actual operating conditions, the thermodynamic model of the solar collector thermoelectric power generation device is established by theoretical derivation; the best cooling conditions is obtained through numerical calculation of thermoelectric generator.
\end{abstract}

\section{Introduction}

At present, the solar energy is a renewable green energy, with an infinite development advantages. But so far solar collector thermoelectric power generation is still in development stage. This article uses the theoretical derivation and experimental determination, graphic simulation and numerical simulation method to establish the thermodynamic model of the thermoelectric power generation device, and the theoretical analysis and experimental verification of the thermoelectric power generation for power generation. In the experiments to explore the performance of solar thermoelectric generator. In this paper, design of device system block diagram as shown in figure 1.

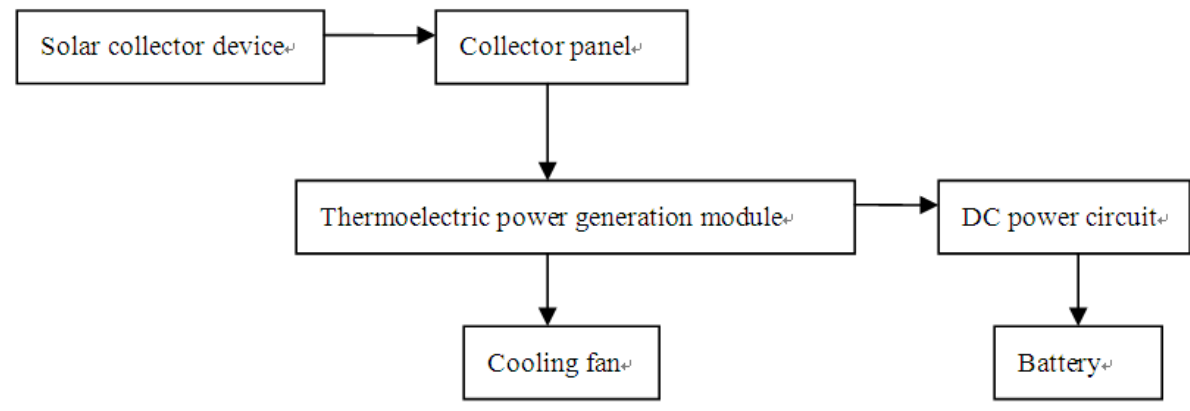

Figure1. Solar collector thermoelectric power generation system block diagram

\section{Analysis of The Performance of Thermoelectric Power Generation}

Fresnel lens concentrate the sun's heat, making thermoelectric generator to produce high temperature hot end; on the other side with forced air convection, the temperature difference in thermoelectric generators at both ends to achieve solar thermal power generation, electricity generated through the DC voltage regulator circuit, to charge the battery.

\section{Thermoelectric Performance Analysis}

\subsection{Performance Parameters}

In actual operation tests, the model used in thermoelectric power generation module is TEG1-199-1.4-0.5.According to the data we get by measuring the different temperature difference between the cold junction and hot junction of thermoelectric power generation chip and the output power under those different conditions, we get the curve of the output power, as Figure 2 shows: 


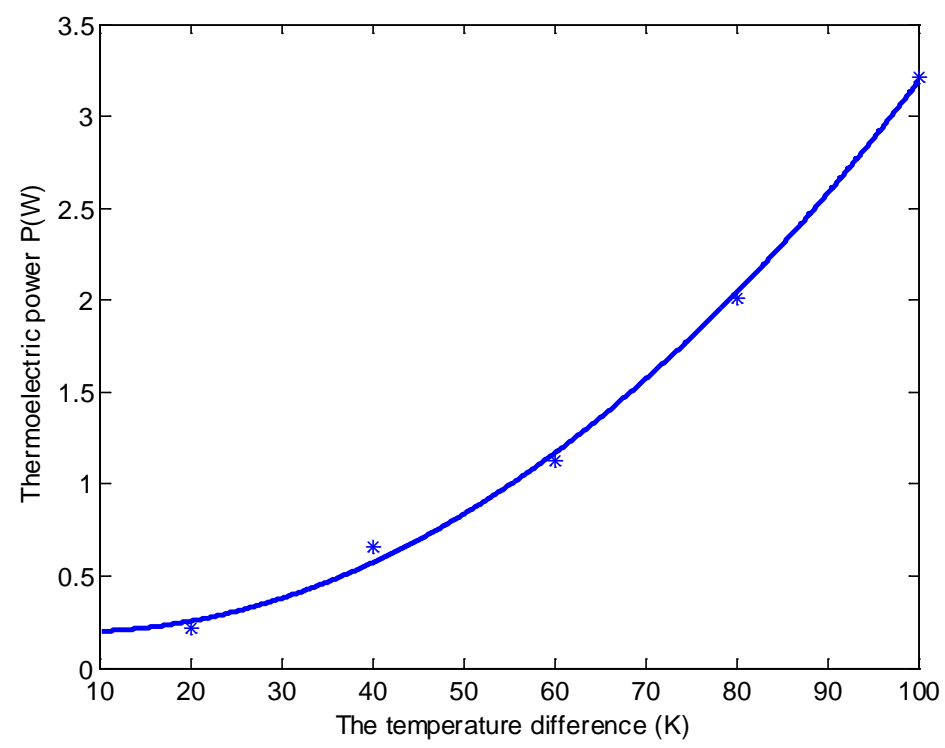

Figure 2. The thermoelectric power with variation of temperature difference

According to the Figure 2, we can draw the conclusion that with the increase of temperature difference between hot and cold junction of the thermoelectric power generation chip, power generation also increase.

\subsection{Thermodynamic Model}

To simplify the problem,we make the following assumptions in the process of modeling:

And the schematic diagram of the temperature difference power generation device just as the Figure 3 shows.

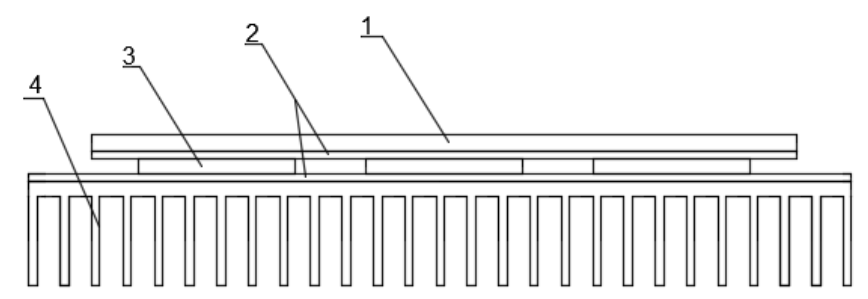

Figure 3 .The schematic diagram of the temperature difference power generation device

1 Aluminum sheet;2 Graphite sheet;3 Thermoelectric power generation sheet;4 Air-cooling fin.

In the practical research, we just analysis the steady heat conduction of the device. The beam through the final lens focus on the heat collecting aluminum sheet, and the heat transfer rate of the aluminum sheet is $\phi$ which is also the total heat that the device absorbs. One part of the heat flows through the thermoelectric power generation chip,then converses into electrical power, of which the power is $P$ and the conversion efficiency is $\eta_{1}$.The other flows from the cold junction, the air-cooling fin, and lost to the surroundings. We use the letter $\phi_{1}$ to describe the heat transfer rate that is used by thermoelectric power generation chip and $\phi_{2}$ to describe the lost part.

So, the total heat transfer rate conducted by aluminum sheet is:

$$
\phi=\frac{T_{1}-T_{2}}{R_{1}}=\frac{T_{1}-T_{2}}{\frac{\delta_{1}}{A_{1} \lambda_{1}}}
$$

Where $A_{1}$ is the area of the aluminum sheet ; $T_{1}$ is the temperature of the aluminum sheet's upper surface ; $T_{2}$ is the temperature of the aluminum sheet's lower surface; $R_{1}$ is thermal resistance of the aluminum sheet ; $\delta_{1}$ is the thickness of aluminum sheet ; $\lambda_{1}$ is the thermal conductivity of the aluminum sheet.

The temperature difference between the hot and cold junction of thermoelectric power generation chip: 
$\Delta T=T_{3}-T_{4}$

Where $T_{3}$ is the temperature of thermoelectric power generation chip's hot junction; $T_{4}$ is the temperature of thermoelectric power generation chip's cold junction.

We can get the relation between the temperature difference and the rate of power generation is:

$P=f(\Delta T)$

The heat that the thermoelectric power generation sheet consumes to generation electricity is:

$\phi_{1}=\frac{P}{\eta_{1}}$

Look up the thermal physical properties of the dry air at standard atmospheric pressure, and get the Nussle number by the feature correlation equation:

$$
\mathrm{Nu}=\frac{0.4637 \mathrm{Re}^{\frac{1}{2}} \mathrm{Pr}^{\frac{1}{3}}}{\left[1+(0.0207 / \mathrm{Pr})^{\frac{2}{3}}\right]^{\frac{1}{4}}}
$$

So the convection heat transfer coefficient is:

$h_{1}=\frac{\lambda_{2}}{l_{2}} \mathrm{Nu}$

Where $\lambda_{2}$ is the thermal conductivity of air; $l_{2}$ is the length of the fin which is along the direction of the air-flow.

The heat flows lost to the surroundings from the air-cooling fin by convection is:

$\phi_{2}=\eta_{\mathrm{f}} \mathrm{h}_{1} \mathrm{~A}_{3}\left(\mathrm{~T}_{4}-\mathrm{t}_{0}\right)$

Where $\eta_{f}$ is efficiency of the fin; $h_{1}$ is the convective heat transfer coefficient of the fin; $A_{3}$ is the total area of the air-cooling fin that exchanges heat with cold air; $T_{4}$ is the temperature of thermoelectric power generation chip's cold junction; $t_{0}$ is the temperature of the cold air.

To simplify the calculation, we just take the area of the fin into account and ignore the area of the root of the fin. To a certain degree ,it is reasonable.

We can get the equation under the law of conservation of energy:

$\phi=\phi_{1}+\phi_{2}$

Through programming, numerical computing, we get the relationship between the air speed $v$ and the rate of power generation ${ }^{p}$ by the thermoelectric power generation chip, as the curve shows in Figure 4:

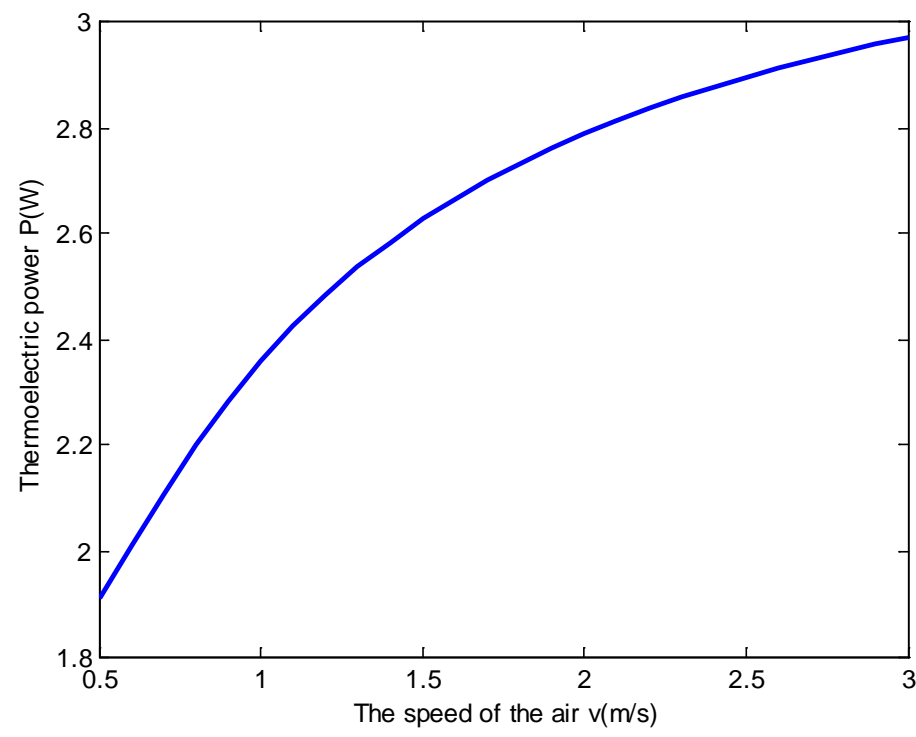

Figure4. The relationship between the rate of power generation ${ }^{p}$ and the air speed $v$

According to the Figure 4, we can get the conclusion that with the increasing of the air speed, the rate of power generation of the thermoelectric power generation chip increases. 


\section{Conclusions}

Having studyed concentrating collectors 、 thermoelectric generators and Cooling method etc. We assembled a set of collector solar thermoelectric power generation device. The thermodynamic model of this device is established by theoretical derivation and we get the optimized air speed $1.8 \mathrm{~m} / \mathrm{s} \sim 2 \mathrm{~m} / \mathrm{s}$ by numerical calculation.

\section{References}

[1]Reng Depeng, Jia Yang, Numerical simulation for operating characteristics of thermogenerator, Spacecraft Engineering, 2008, 17(4):56-61(in Chinese).

[2]Jia Lei, Chen Zeshao, Research on thermal conductivity of thermoelectric generator, Acta Energies Solaris Sinica, 2004, 25(6):816-819

[3]Xuan X C, Li D. Optimization of a combined thermionic-thermoelectric generator, Journal of Power Sources, 2003, 115:167-170

[4]Riffat S B,Xiaoli Ma, [J], Applied Thermal Engineering,2003,23(8);913-935. 